\title{
Small losses, big gains with microRNAs
}

A new study in mice shows that heterozygous loss of Dicer, a crucial component of the microRNA (miRNA) processing machinery, could potentially explain the global decrease in miRNA expression often seen in tumours. Homozygous loss of Dicer1 results in embryonic lethality, so targeted deletion of this gene has been investigated. Mice were engineered to express a floxed oncogenic Kras transgene $\left(L S L-K r a s^{G 12 D}\right)$ that was activated in the lung by inhalation of an adenovirus containing the Cre recombinase. Those mice with either heterozygous or presumed homozygous loss of a floxed Dicer1 gene were found to have increased tumour burden compared with wild-type Dicer 1 mice. However, the heterozygous Dicer 1 mice were found to have the poorest survival rates. Similar results were found in a $\mathrm{Kras}^{G 12 D}$-induced sarcoma model.

Analyses of the $\mathrm{Kras}^{G 12 D}$-driven tumours that arose in animals that were heterozygous and homozygous for Dicer 1 indicated that the 'homozygous' tumours did not show the loss of both alleles. The tumours that arose in mice in which both Dicer 1 alleles were floxed all had incomplete recombination, and one allele was still being expressed. This indicates that complete loss of Dicer1 expression is selected against. Indeed, enforced Dicer 1 homozygosity resulted in impaired tumour growth. These results indicate that Dicer1 is a haploinsufficient tumour-suppressor gene.

Analyses of DICER1 loss in human tumours using data from The Cancer Genome Project and The Cancer Genome Atlas indicated that haploinsufficiency of DICER 1 is relatively common in a variety of tumour types, and that loss of both alleles is not evident. The list of haploinsufficient tumoursuppressor genes is increasing, and the authors warn that genome-wide studies need to consider evidence for haploinsufficiency as well as the traditional loss of tumour-suppressor gene expression by mutation and loss of heterozygosity.

Nicola McCarthy, Chief Editor, Nature Reviews Cancer

ORIGINAL RESEARCH PAPER Madhu, S. K. et al. Dicer 1 functions as a haploinsufficient tumour suppressor. Genes Dev. 10 Nov 2009 (doi 10.1101 / gad.1848209)

FURTHER READING Croce, C. M. Causes and consequences of microRNA dysregulation in cancer. Nature Rev. Genet. 10, 704-714 (2009) | The Cancer Genome Project: http://www.sanger. ac.uk/genetics/CGP | The Cancer Genome Atlas: http://cancergenome.nih.gov 